\title{
A User-Centric Frequency Reuse in Non-full Interference Cellular Networks
}

\author{
Mohammadreza Mardani*, Philippe Mary*, Jean-Yves Baudais ${ }^{\dagger}$ \\ ${ }^{*}$ Univ. Rennes, INSA, IETR, Rennes, France \\ ${ }^{\dagger}$ IETR, CNRS, Rennes, France \\ Email: Mohammadreza.Mardani-Varmazyar@insa-rennes.fr
}

\begin{abstract}
Inspired by the subject of performance enhancement of users located at the edge of cells in cellular networks, this paper studies a centralized frequency reuse scheme depending on the link quality of users within the Voronoi cell of the serving base station (BS) for the homogeneous Poisson point process (PPP) network. To quantify how much a user experiencing performance degradation, we consider that each cell is partitioned into multiple regions based on the strength of the signal to interference ratio. In our model, a given resource block (RB) is allocated to one user in a cell and cannot be shared by another user in this cell. The RB is divided into multiple subchannels and the user of interest uses only one of the multiple sub-channels, the mapping depending on the region in which the user belongs, letting a part of the RB unused to reduce the interference to the other cells. This scenario implies that the interfering set of BS depends on the coverage probability of the typical user. We tightly approximate the interfering BS set as a thinned version of the original PPP depending on the probability of coverage of each type of user. Results show that the scheme increases the network's global coverage probability and enhances spectral efficiency by comparing the known fractional frequency reuse scheme.
\end{abstract}

Index Terms-User classification, Coverage probability, Spectral efficiency, Poisson point process.

\section{INTRODUCTION}

\section{A. Motivation}

In modern cellular networks, the quality of service is correlated to relative signal strength received from serving base station (BS) and all other interfering BS through the so-called signal to interference ratio (SIR), which in turn depends on the network geometry and channel conditions. In a full interference network, users near the cell boundary are known to experience a low SIR [1]. However, in a nonfull interference networks, locating close to cell edge does not necessarily lead important performance degradation because all BS are not active in the same resource [2].

To help users who are more vulnerable to interference, the frequency reuse technique allows reducing the interference by not allocating the same frequencies to neighboring cells. A derivative of that idea is to classify users to cell center/edge user class and apply a location-based approach to assign different sub-bands to each type [3]. However, this is purely geometric and hence does not consider the SIR experienced by the user, that may be a good measure in the full interference scenario, but the SIR can be temporally larger in the non-full interference networks at a point located farther from the BS than the nearest one. In the downlink case, the non-full interference network is a network where all BS do not contribute to the interference received by a user in a given resource block $(\mathrm{RB})$.

In this paper, a cell is divided into $N+1$ classes, and a typical user belongs to a certain class depending on its SIR. Moreover each class has its proper sub-band that may be used by the nearest interfering cell but only by the same type of user in that interfering cell. In our model, a user is classified to be a type- 1 when its SIR is larger than a threshold, a user is type-2 if its SIR is less than the first-class threshold and larger than the second class one, and so on. A user type$N+1$ has the weakest SIR and is in outage from the network. This user-centric model allows to quantify key performances, i.e. the coverage probability and the spectral efficiency (SE) in the non-full interference context, based on the user type. We show that the BS activity is driven by the user position within each cell. In this case, user classification leads to BS classification, which means that the density of the interfering BS set is correlated with the user position in the interfering cells and depends on the location of the user where the SIR is measured. However, it is shown that the correlated interfering scenario can be estimated as a thinning process: the original PPP is split into $N+1$ thinned complementary processes and we quantify the thinning factor for each class.

\section{B. Related Work}

User classification schemes mainly focus on two aspects: the location-based [2]-[6], and the SIR based classification [7]-[9]. However, in classical full interference networks, since all BS are active, both aspects lead to similar conclusions on average and a user located at the cell border undergoes severe performance degradation. For instance, in [4], [5], [10], the ratio of the distance between the typical user and the serving BS to the distance between the typical user to the nearest interfering $\mathrm{BS}$ is computed. If the ratio is larger than a threshold, then the user is a cell edge user; otherwise, it is a cell center user. Based on this kind of classification, base station cooperation techniques have been investigated to enhance the cell edge user coverage [2], [3], [6]. In [7], the whole frequency band has been divided into cell center and cell edge frequency sub-bands to improve the cell edge user coverage using spectrum access techniques such as fractional and soft frequency reuse. In [8], the authors used the instanta- 
neous SINR based classification and got an approximation of the coverage probability of the typical cell edge user for PPPmodeled 3-tier heterogeneous networks. Within this general direction, the authors in [10] used the location-based cell center/edge user classification and derived the moments of the meta distribution under non-orthogonal/orthogonal multiple access techniques. All these previous works have been done with full interference assumption, i.e., considering that all $\mathrm{BS}$, or a fraction of them but non-related to the coverage probability, act as interferers to the typical user whatever the bandwidth it uses. However, these are not always a good measure because, in practice, there are many inactive BS in the network. In [2], the authors proposed a new definition for the cell edge users in small cell deployments based on the nearest active base stations (non-empty cells) to evaluate the user performance degradation in practice. However, it does not consider the received SIR that contains the relevant statistic of the communication performance, and which can correlate the activity of a BS with the coverage probability of its user. The BS activity depends on the coverage probability that lead to a thinning over the original PPP in a given resource block that in turn leads to a non-full interference situation. This is what is called a user-centric thinning that has been explored in [2] to analyze the performance of cell-edge users in small cell networks. In a previous work [9], a SIRdependent non-full interference model has been introduced in which the given resource is divided into two sub-channels that are used exclusively according to whether the user is a cell center or cell edge user and lets a part of the resource unused to reduce the interference. The central coverage probability has been found to be the solution of a fix point equation.

In this paper, we extend the work in [9] to $N+1$ user classes and key performance metrics, i.e. the coverage probability and the SE, in a non-full interference context are tackled. In our work, the non-full interference setting refers to the fact that the interference in a given sub-channel depends on the coverage probability of the user type according to the sub-channel considered. Section II presents our system model. Our main results are stated in Theorems 1 and 2 in Section III. Section IV presents the numerical results and conclusions are drawn in Section V.

Notations: Random variables are denoted in capital font while their realizations remain in small font. Moreover, $\mathbb{P}(\cdot)$ and $\mathbb{E}[\cdot]$ are the probability and the expectation operators. $1(\cdot)$ is the indicator function.

\section{SySTEM Model}

Let us consider an OFDMA-based single-tier downlink cellular network where the BS deployment is modeled as an homogeneous PPP $\Phi \subset \mathbb{R}^{2}$ with density $\lambda$. The users are associated with their nearest BS, and all BS and users are equipped with a single antenna. Similarly to the works in [9], [11], only one randomly chosen user is considered to communicate with its BS on a given time-frequency resource. We assume that users are experiencing Rayleigh fading channel. In each cell, the typical user is a type- $k$ user according to the relative value of its SIR w.r.t. some thresholds that, in turn, depends on the location of others BS and on the channel conditions. Fig. 1 illustrates one realization of a PPP network for two types of served users, with only one user per BS. The BS colored in green, red and black represent the active BS for users of type-1, type-2 and outage users respectively. An RB is allocated to one user in each cell. Contrarily to $4 \mathrm{G}$ or $5 \mathrm{G}$ systems, the RB is divided into multiple sub-channels. A sub-channel $k$ is used by the type- $k$ user. However, since the entire RB is dedicated to a given type of user, BS cannot allocate the remaining part of the RB to another user in the cell. This setting leads to a non-full load context for interference in each sub-channel because only a part of the RB is used in a given cell.

In that context, let us consider the typical BS at $X_{0}$ and divide the interfering BS set $\Phi \backslash\left\{X_{0}\right\}$ into $N+1$ complementary subsets $\Phi_{k}$ such that $\Phi \backslash\left\{X_{0}\right\}=\cup_{k=1}^{N+1} \Phi_{k}$ and $\Phi_{k} \cap \Phi_{l}=\emptyset$ for all $k \neq l . \Phi_{k}$ is the subset gathering the BS that are serving type- $k$ users across the network and hence $\Phi_{N+1}$ is the set of BS that have the typical user in outage. Let $X_{i}$ be the position of the BS $i$ and $\operatorname{SIR}_{i}^{k}$ the SIR experienced by a randomly selected user in the cell $i$ over the sub-channel $k$. The subset $\Phi_{k}$ for all $1 \leq k \leq N$ is

$$
\Phi_{k} \triangleq\left\{X_{i} \mid \operatorname{SIR}_{i}^{k} \geq \theta_{k}, X_{i} \in \Phi_{k-1}^{!}\right\},
$$

and $\Phi_{N+1}=\Phi_{N}^{!}$, where $\Phi_{k}^{!}=\Phi \backslash\left\{\cup_{m=1}^{k} \Phi_{m}\right\}$ for all $k$ and real numbers $\left\{\theta_{k}\right\}_{1}^{N} \geq 0$ are the SIR classification thresholds. Let $\operatorname{SIR}_{0}^{k}$ be the received SIR by the typical user of the BS $X_{0}$ in the subchannel $k$, then

$$
\operatorname{SIR}_{0}^{k}=\frac{H_{0,0}^{k} R_{0}^{-\alpha}}{\sum_{X_{i} \in \Phi \backslash\left\{X_{0}\right\}} H_{0, i}^{k} R_{i}^{-\alpha} 1\left(X_{i} \in \Phi_{k}\right)},
$$

where $R_{i}$ is the distance between the BS $i$ and the typical type- $k$ user, $H_{0, i}^{k}$ is the channel gain between the typical user located at the origin and the BS $i$ in sub-channel $k$, and $\alpha>2$ is the path loss exponent. Since separated frequency sub-bands are allocated to different user types, they do not experience the same instantaneous channel gain but $H_{0, i}^{k}$ are independent and identically exponentially distributed (i.i.e.d.). The indicator function in (2) ensures that the typical user experiences interference only from BS that serve other type- $k$ users. This model enlightens the fact that this is an interdependent thinning process, and we obtain in this paper that it can be tightly approximated by a thinning process on BS PPP by a factor depending on the coverage probability.

\section{Performance Metrics}

In this section, we first analyze the coverage probability of a typical randomly located user in the non-full interference network. Next, the conditional coverage probability of each user is investigated. Finally, to fairly evaluate the proposed method, the SE of the network is studied. 


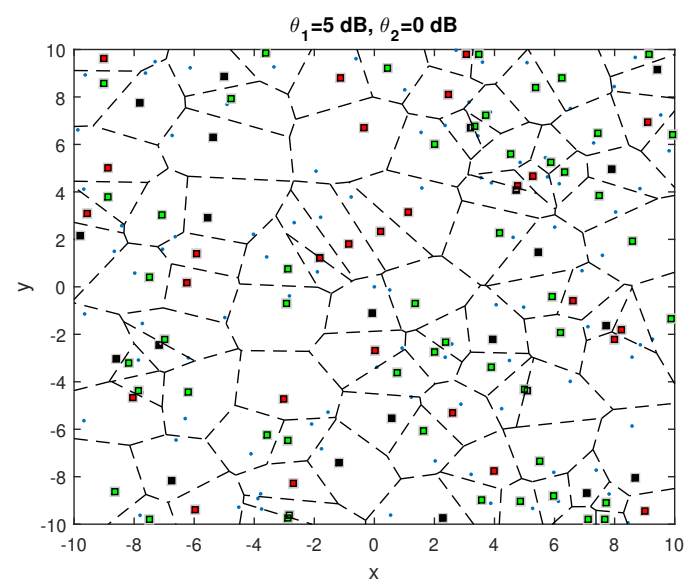

Figure 1. PPP deployment for BS with a randomly selected user (blue dots) in each cell.

\section{A. Coverage Probability}

In interference-limited wireless networks, the standard coverage probability describes the probability that the SIR of the typical link exceeds a threshold [11]. In our model, we have a set of thresholds $\left\{\theta_{k}\right\}_{1 \leq k \leq N}$ to successfully demodulate and decode the received signal. Then, the typical user placed at the origin is covered on downlink if one of the subsequent events occurs:

$$
\left\{X_{0} \in \Phi_{k}\right\}=\left\{\operatorname{SIR}_{0}^{k} \geq \theta_{k}, X_{0} \in \Phi_{k-1}^{!}\right\}, k=1, \ldots, N .
$$

Referring to the law of total probability, the coverage probability of the typical user is as follows.

Theorem 1. The coverage probability $p_{c}$ of a randomly located typical user in a non-full interference network with $N$ type of covered users is given by

$$
p_{c}\left(\left\{\theta_{k}\right\}, \alpha, \lambda\right)=\sum_{k=1}^{N} p_{k}
$$

where $p_{k}$ is well estimated with a fixed point equation as

$$
p_{k}=\int_{0}^{\infty} e^{-v\left(1+p_{k} \rho\left(\theta_{k}, \alpha\right)\right)} \prod_{i=1}^{k-1}\left(1-e^{-p_{i} \rho\left(\theta_{i}, \alpha\right) v}\right) \mathrm{d} v
$$

and $\rho(\theta, \alpha)=\theta^{\delta} \int_{\theta^{-\delta}}^{\infty} \frac{1}{1+x^{1 / \delta}} \mathrm{d} x$, where $\delta=2 / \alpha$.

Proof. See Appendix A.

This expression for coverage probability does not depend on BS density $\lambda$ and is calculated using the system parameters, i.e. path loss exponent $\alpha$, SIR thresholds $\left\{\theta_{k}\right\}_{1 \leq k \leq N}$, and as expected it goes to 1 for all $\theta_{k} \rightarrow 0$ and 0 for all $\theta_{k} \rightarrow \infty$.

Furthermore, the conditional coverage probability $\mathcal{P}_{k}$ of type- $k$ user in the non-full interference network is defined as

$$
\mathcal{P}_{k}\left(\left\{\theta_{k}\right\}, \alpha, \lambda\right) \triangleq \mathbb{P}\left(\operatorname{SIR}_{0}^{k} \geq \theta_{k} \mid X_{0} \in \Phi_{k-1}^{!}\right) .
$$

Corollary 1. Conditioning on $X_{0} \in \Phi_{k-1}^{!}$, the probability that $\operatorname{SIR}_{0}^{k}$ satisfies a threshold $\theta_{k}$ is given by

$$
\mathcal{P}_{k}\left(\left\{\theta_{k}\right\}, \alpha, \lambda\right)=\frac{p_{k}}{\int_{0}^{\infty} e^{-v} \prod_{i=1}^{k-1}\left(1-e^{-p_{i} \rho\left(\theta_{i}, \alpha\right) v}\right) \mathrm{d} v}
$$

Proof. Starting with (6) and using the Bayes rule, we have Conditioned on $X_{0} \in \Phi_{k-1}^{!}$, the coverage probability of type-k user $\mathcal{P}_{k}$ is

$$
\begin{aligned}
\mathcal{P}_{k}\left(\left\{\theta_{k}\right\}, \alpha, \lambda\right) & =\mathbb{P}\left(\operatorname{SIR}_{0}^{k} \geq \theta_{k} \mid X_{0} \in \Phi_{k-1}^{!}\right) \\
& =\frac{\mathbb{P}\left(\operatorname{SIR}_{0}^{k} \geq \theta_{k}, X_{0} \in \Phi_{k-1}^{!}\right)}{\mathbb{P}\left(X_{0} \in \Phi_{k-1}^{!}\right)} .
\end{aligned}
$$

The rest of the proof straightforwardly follows from Theorem 1.

The expressions obtained in (4) and (7) are not tractable to find a closed-form expression for the typical user coverage probability, except for the coverage probability of the typical type-1 (cell interior) user:

$$
\mathcal{P}_{1}\left(\theta_{1}, \alpha, \lambda\right)=p_{1}=\frac{\sqrt{1+4 \rho\left(\theta_{1}, \alpha\right)}-1}{2 \rho\left(\theta_{1}, \alpha\right)} .
$$

In particular, when $\alpha=4$ (high path-loss condition), we have $\rho(\theta, 4)=\sqrt{\theta}\left(\frac{\pi}{2}-\arctan \left(\frac{1}{\sqrt{\theta}}\right)\right)$.

In the single-user type case, i.e. $N=1$, if $\theta_{1}=1(0$ $\mathrm{dB}$ ), the coverage probability of a typical user in (4) is equal $2 \pi^{-1}(\sqrt{1+\pi}-1)=0.66$ which gains $0.7 \mathrm{~dB}$ rather than the coverage probability of a typical user in the classical full interference network given in [11]. As the coverage does not take into account the resource used, we need another measure to evaluate and compare the proposed method fairly. So, we investigate the SE as follows.

\section{B. Spectral Efficiency}

Considering the system setting of [11], where only one type of user is considered, i.e. the user is covered, the average rate per RB for the typical user is $\mathbb{E}[\ln (1+\mathrm{SINR})]$. In our case, a given RB is divided into $N$ sub-channels each assigned to its corresponding user type and the conditional SE in nats $/ \mathrm{s} / \mathrm{Hz}$ of the typical user of type- $k$ is defined as

$$
\mathcal{R}_{k} \triangleq \omega_{k} \mathbb{E}\left[\ln \left(1+\operatorname{SIR}_{0}^{k}\right) \mid X_{0} \in \Phi_{k}\right],
$$

where $0 \leq \omega_{k} \leq 1$ and $\sum_{k=1}^{N} \omega_{k}=1$.

Theorem 2. Conditioning on the typical user being of type- $k$, the spectral efficiency $\mathcal{R}_{k}$ is given by

$$
\mathcal{R}_{k}=\frac{\omega_{k}}{p_{k}}\left(p_{k} \ln \left(1+\theta_{k}\right)+\int_{\theta_{k}}^{\infty} g_{k}(z) \mathrm{d} z\right),
$$

where

$$
g_{k}(z)=\frac{1}{1+z} \int_{0}^{\infty} e^{-v\left(1+p_{k} \rho(z, \alpha)\right)} \prod_{i=1}^{k-1}\left(1-e^{-p_{i} \rho\left(\theta_{i}, \alpha\right) v}\right) \mathrm{d} v .
$$

Proof. See Appendix B.

Hence, the overall SE of the typical user in the non-full interference network is $\mathcal{R}=\sum_{k=1}^{N} p_{k} \mathcal{R}_{k}$. 


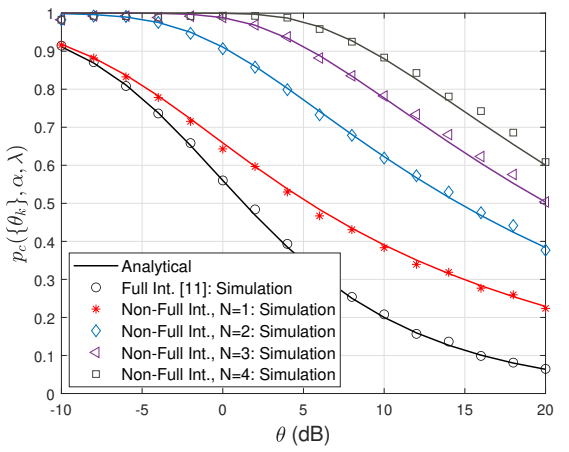

(a)

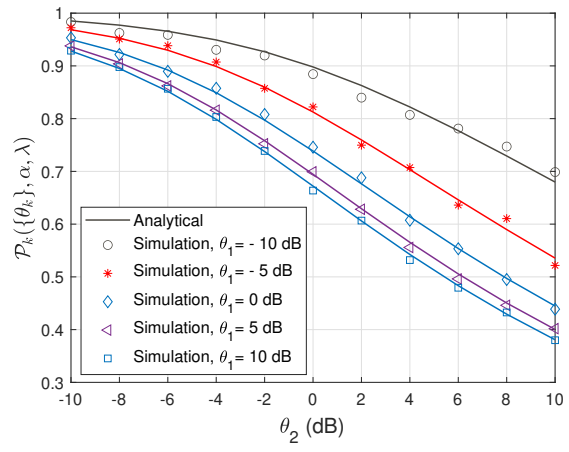

(b)

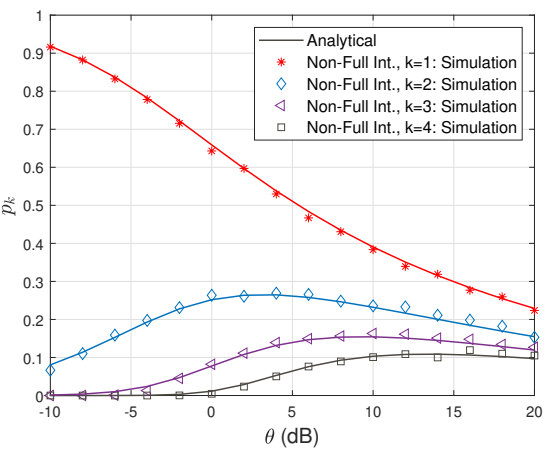

(c)

Figure 2. (a) Coverage probability, (b) Conditional coverage probability for $k=2$ users case, (c) Probability of being type-k user.

\section{Bandwidth Allocation}

In this part, we investigate two strategies to allocate the available bandwidth among $N$ class of users to have the maximum overall spectral efficiency or fair bandwidth allocation among different user types. To maximize the overall spectral efficiency, the maximization problem can be written as

$$
\begin{gathered}
\max _{\left\{\omega_{k}\right\}_{1 \leq k \leq N}} \sum_{k=1}^{N} \omega_{k}\left(p_{k} \ln \left(1+\theta_{k}\right)+\int_{\theta_{k}}^{\infty} g_{k}(z) \mathrm{d} z\right) \\
\text { subject to: } \quad \sum_{k=1}^{N} \omega_{k}=1, \omega_{k} \geq 0 .
\end{gathered}
$$

The optimal solution of (13) is simply given by $\omega_{k}=1$ for the index $k$ corresponding to the largest value of $p_{k} \ln \left(1+\theta_{k}\right)+$ $\int_{\theta_{k}}^{\infty} g_{k}(z) \mathrm{d} z$, which is function of the set of the classification thresholds, and $\omega_{k}=0$ otherwise. However, this strategy does not allow fairness among users and it can be preferable to share the RB among all the covered users, i.e. $\omega_{k}>0$ for all $k$. Two policies can be followed.

1) Fixed bandwidth partitions. This model assigns a real value in $(0,1)$ to $\omega_{k}$, based on the quality of service required by the type- $k$ user. The simplest partition policy, which does not need any other information from the system, is an equal partitioning method where the RB is equally divided into $N$ equal sub-channels, i.e. $\omega_{k}=1 / N$. In this case, the overall rate of the given typical user is

$$
\mathcal{R}=\frac{1}{N} \sum_{k=1}^{N}\left(p_{k} \ln \left(1+\theta_{k}\right)+\int_{\theta_{k}}^{\infty} g_{k}(z) \mathrm{d} z\right) .
$$

2) Adaptive bandwidth partitions. The available bandwidth is shared among the users according to the SIR distribution and traffic load. In a regular grid network, the frequency reuse scheme relies on a geometry-based policy to allocate a set of contiguous bandwidth chunks among cell center and cell edge users which is proportional to the square of the ratio of the interior and the cell radius [7]. In PPP models, geometric foreknowledge for chunk allocation is not employed and, instead, the allocation can be made based on the SIR distribution [7]. Hence, in the SIR-proportional model, we have $\omega_{k}=p_{k}$ and the overall rate is

$$
\mathcal{R}=\sum_{k=1}^{N}\left(p_{k}^{2} \ln \left(1+\theta_{k}\right)+p_{k} \int_{\theta_{k}}^{\infty} g_{k}(z) \mathrm{d} z\right)
$$

\section{Numerical Results}

To verify the analytical results, we conduct simulations in a PPP network. The coverage probability and the SE are evaluated at the typical user for 100,000 realizations of the network. We consider an interference-limited scenario with $\alpha=4$. In each realization, the BS locations are generated as a PPP of unit intensity in an area of $[-10,10]^{2}$, and user density is considered large enough to have at least one randomly chosen user per cell.

Fig. 2(a) shows the simulation and the analytical results for the coverage probability (4) for a typical user when $N \in$ $\{1,2,3,4\}$, under non-full and full interference scenarios when a unique $\theta$ is considered, i.e. the target threshold is the same for all user classes. The non-full interference strategy induced by the user-centric RB allocation according to the type of users, leads to a larger coverage probability when $N$ increases. The analytical derivations are relatively close to the simulations especially for small values of threshold. However, the mismatch increases as the threshold and $\mathrm{N}$ increase. The gap comes from the use of the independence assumption in the derivation of Theorem 1. Moreover the estimation of the type- $k$ interfering set of BS by simulation is an iterative process that is sensitive to the threshold value and requires a lot of iterations when $\theta$ is large. Nonetheless, this approach fits when the threshold $\theta$ is not too large to ensure enough active BS for large scale network approximation in simulation.

In Fig. 2(b), the type-2 user conditional coverage probability $\mathcal{P}_{2}$ in (7) is plotted versus $\theta_{2}$ for specific type- 1 target thresholds $\theta_{1}=\{-10,-5,0,5,10\} \mathrm{dB}$. As seen in the figure, when $\theta_{1}$ decreases, the number of active BS in the type2 class, i.e. interfering BS, decreases and consequently the conditional coverage probability of the typical type- 2 user increases when $\theta_{1}$ decreases. 


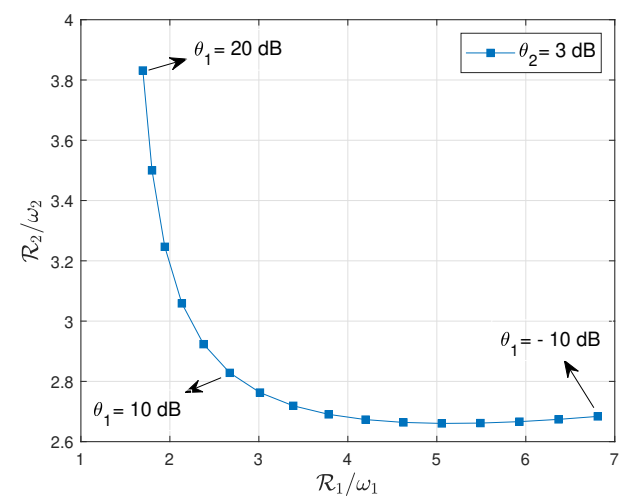

Figure 3. Type-1 and type-2 SE trade-off.

Fig. 2(c) shows the probability $p_{k}$ in (5) that the typical user be a type- $k$ user for $k \in\{1,2,3,4\}$. From the figure, it can be seen that when increasing the unique target threshold $\theta$, the probability $p_{1}$ decreases, but $p_{2}, p_{3}$ and $p_{4}$ increase and then decrease after a specific threshold value.

Fig. 3 represents the trade-off between the spectral efficiencies of type- 1 and type- 2 user classes regardless of the bandwidth allocation strategy and evaluated with (11). The tradeoff curve varies versus the value of $\theta_{1}=[-10,20] \mathrm{dB}$ when the threshold of type- 2 has specific value, i.e. $\theta_{2}=3 \mathrm{~dB}$. The type-2 spectral efficiency increases with $\theta_{1}$, since the number of type- 2 user increases, and vice versa for the type- 1 user spectral efficiency.

Fig. 4 shows the overall spectral efficiency in the case of two user types under SIR-proportional bandwidth allocation strategy [7]. From the figure, it can be found that for the specific values of $\theta_{1}$, the overall spectral efficiency increases and decreases after $6 \mathrm{~dB}$, because the number of the type- 2 users decreases and it is not compensated by the gain of being closer to the BS.

Fig. 5 represents the overall spectral efficiency for $N \in$ $\{1,2,3\}$ in the non-full interference network versus the type1 user target threshold $\theta_{1}$ when $\theta_{2}=3 \mathrm{~dB}$ and $\theta_{2}=1 \mathrm{~dB}$. The SE of different types of users are compared under fixed equal partitioning (14) and adaptive SIR-proportional (15) strategies. When $\theta_{1}$ increases, the total SE under SIRproportional policy decreases and it increases under equal partitioning. Moreover, the total SE is compared with the fractional frequency reuse (FFR) technique with reuse factor $\Delta$ presented in [11], under full interference network, i.e. only one type of user. Since in SIR-proportional policy, the typical user benefits from a fraction of resources that depends on the SIR, and suffers from the same fraction of the interference, it has higher SE than FFR with $\Delta=1, \Delta=2$ and $\Delta=3$, respectively.

\section{CONCLUSion}

This paper has presented a complete characterization of the downlink coverage probability and spectral efficiency of a typical user in a non-full interference homogeneous

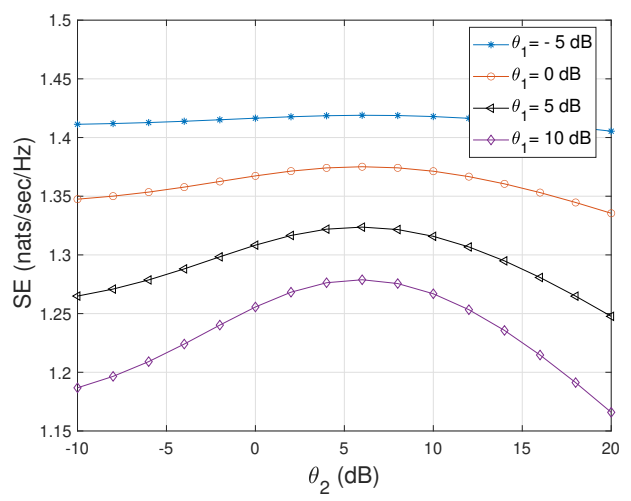

Figure 4. Overall SE for $k=2$ users case.

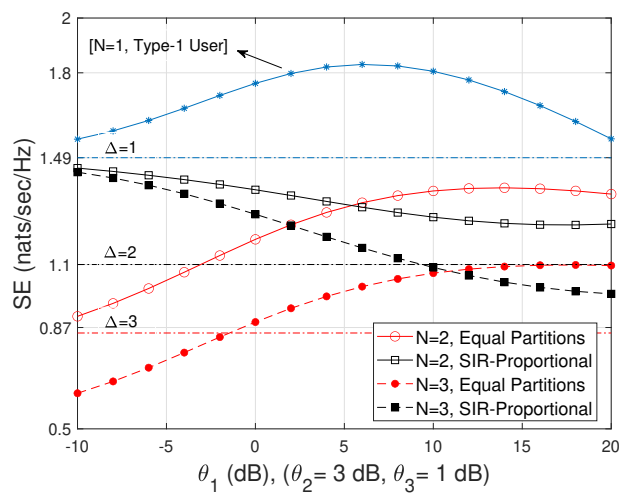

Figure 5. SE under equal and SIR-proportional partitioning strategies.

PPP network. Semi closed-form expressions of performance metrics based on the received SIR level for the typical user have been presented. The results show that the user-centric resource allocation approach outperforms the conventional FFR approach, which is BS-centric. In further works, we intend to investigate system performance when resource allocation is performed according to the number of users in the cells which implies correlations among two PPPs.

\section{ACKNOWLEDGEMENT}

This work has been partially funded by the Region Bretagne, France, under the project ARED CEREAL.

\section{APPENDIX}

\section{A. Appendix A: Proof of Theorem 1}

Based on the law of total probability, the coverage probability $p_{c}$ of the typical user is the probability that it satisfies the required condition of at least one of the $N$ type user classes in (1). Hence, the coverage probability can be expressed as $p_{c}=\sum_{k=1}^{N} p_{k}$ where $p_{k}$ is the probability of being a type- $k$ user, i.e.

$$
p_{k}=\mathbb{P}\left(X_{0} \in \Phi_{k}\right)=\mathbb{P}\left(\operatorname{SIR}_{0}^{k} \geq \theta_{k}, X_{0} \in \Phi_{k-1}^{!}\right),
$$


Conditioning on $R_{0}$ the two events in (16) are independent and it can be written as

$$
p_{k}=\int_{0}^{\infty} \mathbb{P}\left(\operatorname{SIR}_{0}^{k} \geq \theta_{k} \mid R_{0}\right) \mathbb{P}\left(X_{0} \in \Phi_{k-1}^{!} \mid R_{0}\right) f_{R_{0}}\left(R_{0}\right) \mathrm{d} R_{0},
$$

where $f_{R_{0}}\left(R_{0}\right)=2 \pi \lambda R_{0} e^{-\pi \lambda R_{0}^{2}}$. The first term in (17) is the probability that the SIR at the typical receiver exceeds $\theta_{k}$ and it follows

$$
\begin{aligned}
& \mathbb{P}\left(H_{0,0}^{k} \geq \theta_{k} R_{0}^{\alpha} \sum_{X_{i} \in \Phi \backslash\left\{X_{0}\right\}} H_{0, i}^{k} R_{i}^{-\alpha} 1\left(X_{i} \in \Phi_{k}\right)\right) \\
& \stackrel{a}{=} \mathbb{E}\left[\exp \left(-\theta_{k} R_{0}^{\alpha} \sum_{X_{i} \in \Phi \backslash\left\{X_{0}\right\}} H_{0, i}^{k} R_{i}^{-\alpha} 1\left(X_{i} \in \Phi_{k}\right)\right)\right] \\
& \stackrel{b}{=} \mathbb{E}\left[\prod_{X_{i} \in \Phi \backslash\left\{X_{0}\right\}}\left(1-\mathbb{E}\left[1\left(X_{i} \in \Phi_{k}\right)\right]\left(1-e^{-\theta_{k} R_{0}^{\alpha} H_{0, i}^{k} R_{i}^{-\alpha}}\right)\right)\right],
\end{aligned}
$$

where (a) comes from the i.i.d. exponential distribution of $H_{0,0}^{k}$ with mean 1 as in [11], (b) comes from averaging over interfering fading channels, the law of total expectation, $\mathbb{E}_{X}[f(X)]=\mathbb{E}_{Y}\left[\mathbb{E}_{X}[f(X) \mid Y]\right]$, the independence of $\left\{\operatorname{SIR}_{i}^{k}\right\}_{1 \leq i \leq N}$, which is a reasonable assumption whose correctness has been verified by simulations, and finally by factoring out $\mathbb{E}\left[1\left(X_{i} \in \Phi_{k}\right)\right]$. By applying the probability generating function (PGFL) [12] of the PPP and identically distributed $\left\{\operatorname{SIR}_{i}^{k}\right\}_{i}$, we have

$$
\mathbb{P}\left(\operatorname{SIR}_{0}^{k} \geq \theta_{k} \mid R_{0}\right)=\exp \left(-2 \pi \lambda p_{k} \rho\left(\theta_{k}, \alpha\right) R_{0}^{2}\right) .
$$

The second term of (17) means that the received SIR by the typical user is less than all $\left\{\theta_{i}\right\}_{i=1}^{k-1}$ and can be derived as

$$
\begin{aligned}
\mathbb{P}\left(X_{0} \in \Phi_{k-1}^{!} \mid R_{0}\right) & =\prod_{i=1}^{k-1} \mathbb{P}\left(\operatorname{SIR}_{0}^{i}<\theta_{i} \mid R_{0}\right) \\
& =\prod_{i=1}^{k-1}\left(1-\mathbb{P}\left(\operatorname{SIR}_{0}^{i} \geq \theta_{i} \mid R_{0}\right)\right) \\
& =\prod_{i=1}^{k-1}\left(1-\exp \left(-2 \pi \lambda p_{i} \rho\left(\theta_{i}, \alpha\right) R_{0}^{2}\right)\right)
\end{aligned}
$$

By putting (19) and (20) in (17) and taking expectation over $R_{0}$, we reach to (5). Also, deconditioning (20) and with $v=$ $\pi \lambda R_{0}^{2}$, we have

$$
\mathbb{P}\left(X_{0} \in \Phi_{k-1}^{!}\right)=\int_{0}^{\infty} e^{-v} \prod_{i=1}^{k-1}\left(1-e^{-p_{i} \rho\left(\theta_{i}, \alpha\right) v}\right) \mathrm{d} v
$$

\section{B. Appendix B: Proof of Theorem 2}

Conditioning on the fact that typical user being of type- $k$, the spectral efficiency $\mathcal{R}_{k}$ is given as

$$
\mathcal{R}_{k}=\omega_{k} \mathbb{E}\left[\ln \left(1+\operatorname{SIR}_{0}^{k}\right) \mid \operatorname{SIR}_{0}^{k} \geq \theta_{k}, X_{0} \in \Phi_{i-1}^{!}\right],
$$

knowing that for a positive random variable $Y, \mathbb{E}[Y]=$ $\int_{u>0} P(Y>u) \mathrm{d} u$, we have

$\mathcal{R}_{k}=\int_{0}^{\infty} \frac{\omega_{k} \mathbb{P}\left(\operatorname{SIR}_{0}^{k} \geq e^{u}-1, \operatorname{SIR}_{0}^{k} \geq \theta_{k}, X_{0} \in \Phi_{i-1}^{!}\right)}{\mathbb{P}\left(\operatorname{SIR}_{0}^{k} \geq \theta_{k}, X_{0} \in \Phi_{i-1}^{!}\right)} \mathrm{d} u$.

From the same approach used in the proof of Theorem 1, it can be written as

$$
\mathcal{R}_{k}=\frac{\omega_{k}}{p_{k}} \int_{0}^{\infty} \mathbb{P}\left(\operatorname{SIR}_{0}^{k} \geq \theta_{k, \text { max }}, X_{0} \in \Phi_{i-1}^{!}\right) \mathrm{d} u,
$$

where $\theta_{k, \max }=\max \left(\theta_{k}, e^{u}-1\right)$, and the term inside the integral can be simplified by dividing the integral bound into $\left[0, \ln \left(1+\theta_{k}\right)\right]$ and $\left[\ln \left(1+\theta_{k}\right), \infty\right]$, Hence, we can rewrite (24) as follow

$$
\begin{aligned}
\mathcal{R}_{k} & =\frac{\omega_{k}}{p_{k}}\left[\int_{0}^{\ln \left(1+\theta_{k}\right)} \mathbb{P}\left(\operatorname{SIR}_{0}^{k} \geq \theta_{k}, X_{0} \in \Phi_{i-1}^{!}\right) \mathrm{d} u\right. \\
& \left.+\int_{\ln \left(1+\theta_{k}\right)}^{\infty} \mathbb{P}\left(\operatorname{SIR}_{0}^{k} \geq\left(e^{u}-1\right), X_{0} \in \Phi_{i-1}^{!}\right) \mathrm{d} u\right] .
\end{aligned}
$$

Using (19) and (20), and applying the change of variable $z=e^{u}-1$, (11) is obtained.

\section{REFERENCES}

[1] M. Kamel, W. Hamouda, and A. Youssef, "Ultra-dense networks: A survey," IEEE Communications Surveys \& Tutorials, vol. 18, no. 4, pp. 2522-2545, 2016.

[2] J. Yoon and G. Hwang, "Distance-based inter-cell interference coordination in small cell networks: stochastic geometry modeling and analysis," IEEE Trans. Wireless Commun, vol. 17, no. 6, pp. 40894103, Jun. 2018.

[3] K. Feng and M. Haenggi, "A location-dependent base station cooperation scheme for cellular networks," IEEE Trans. Commun., vol. 67 no. 9, pp. 6415-6426, Sep. 2019.

[4] P. Mankar, G. Das, and S. Pathak, "Load-aware performance analysis of cell center/edge users in random hetnets," IEEE Trans. Veh. Technol., vol. 67, no. 3, pp. 2476-2490, Mar. 2018.

[5] C. Skouroumounis, C. Psomas, and I. Krikidis, "Heterogeneous fdmm-wave cellular networks with cell center/edge users," IEEE Trans. Commun., vol. 67, no. 1, pp. 791-806, Jan. 2019.

[6] H. Wang, K. Huang, and T. A. Tsiftsis, "Base station cooperation in millimeter wave cellular networks: Performance enhancement of celledge users," IEEE Trans. Commun., vol. 66, no. 11, pp. 5124-5139, Nov. 2018.

[7] T. D. Novlan, R. K. Ganti, A. Ghosh, and J. G. Andrews, "Analytical evaluation of fractional frequency reuse for ofdma cellular networks," IEEE Trans. Wireless Commun., vol. 10, no. 12, pp. 4294-4305, Dec. 2011.

[8] H. Zhuang and T. Ohtsuki, "A model based on poisson point process for analyzing mimo heterogeneous networks utilizing fractional frequency reuse," IEEE Trans. Wireless Commun., vol. 13, no. 12, pp. 6839-6850, Dec. 2014.

[9] M. Mardani, P. Mary, and J.-Y. Baudais, "A tractable model for coverage in non-full interference cellular networks with cell center/edge users," in International Workshop on Signal Processing Advances in Wireless Communications, Atlanta, GA, USA, May 2020, pp. 1-5.

[10] P. D. Mankar and H. S. Dhillon, "Downlink analysis of noma-enabled cellular networks with 3gpp-inspired user ranking," IEEE Trans. Wireless Commun., vol. 19, no. 6, 2020.

[11] J. G. Andrews, F. Baccelli, and R. K. Ganti, "A tractable approach to coverage and rate in cellular networks," IEEE Tran. Commun., vol. 59, no. 11, pp. 3122-3134, Nov. 2011.

[12] S. N. Chiu, D. Stoyan, W. S. Kendall, and J. Mecke, Stochastic geometry and its applications. John Wiley \& Sons, 2013. 